\title{
Correlates of In-Law Conflict and Intimate Partner Violence Against Chinese Pregnant Women in Hong Kong
}

Ko Ling Chan

\author{
Agnes Tiwari \\ Daniel Fong \\ University of Hong Kong \\ Wing Cheong Leung \\ Kwong Wah Hospital, Hong Kong \\ Douglas A. Brownridge \\ University of Manitoba, Canada
}

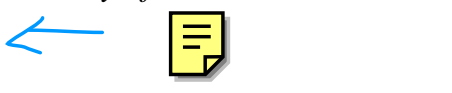

This study examines correlates of in-law conflict with intimate partner violence (IPV) against pregnant women in a cohort of Chinese pregnant women who visited antenatal clinics in Hong Kong. This was a territory-wide, cross-sectional study of 3,245 pregnant women recruited from seven hospitals in Hong Kong. Participants were invited to complete the Chinese Abuse Assessment Screen and a demographic questionnaire. About $9 \%$ of the pregnant women reported having been abused by their partners in the preceding year. In-law conflict was the characteristic most significantly associated with preceding-year abuse against pregnant women, after controlling for covariates. Findings underscore the need to obtain information on in-law conflict as a risk factor for IPV. In-law conflict should be included in the assessment of risk for IPV. For the prevention of IPV, family-based intervention is needed to work with victims as well as in-laws.

Keywords: in-law conflict; intimate partner violence; Chinese; pregnancy and violence

$\mathrm{V}$ iolence against women is a serious social problem in Chinese societies. The prevalence of spousal violence ranges from $1.8 \%$ ( $\mathrm{W}$. Leung, $\mathrm{Ng}$, Leung, \& Ho, 2003) to 50.0\% (Yick, 1999), depending on the samples and instruments used. In the studies of pregnant women in Hong Kong and 
women attending an outpatient gynecological clinic in Fuzhou, China, it was found that $17.9 \%$ and $43.0 \%$, respectively, reported a history of abuse (W. Leung, Leung, Lam, \& Ho, 1999; Xu et al., 2005). The existing approach to studying risk factors specific to intimate partner violence (IPV) in Chinese societies mainly involves determining whether universal risk factors are also applicable to the Chinese. However, these factors do not adequately address culture- and society-specific characteristics of Chinese societies. Fernandez (1997) argued that unlike the typical domestic violence scenario in the West that usually involves a lone man battering a lone woman, violence against women in Asia usually involves the husband's family, particularly senior women such as the wife's mother-in-law. Other studies in Indian, Indo-Fijian, and Taiwanese cultures (Counts, Brown, \& Campbell, 1999) have similarly found that the mother-in-law plays a role in wife beating and that it is probably common in collectivist societies in general.

The mother-in-law could be a perpetrator of violence against women (Ramanathan, 1996; Rianon \& Shelton, 2003) even against pregnant women (Dasgupta, 2000; $\mathbb{W}$ C Leung et al., 1999). A very recent study of South Asians in the United States found a significant relationship between IPV and emotional abuse by in-laws (Raj, Livramento, Santana, Gupta, \& Silverman, 2006). Related, conflict with a mother-in-law could be a risk factor for the wife's postnatal depression (Lee, Yip, Leung, \& Chung, 2004) and for the husband's violence (Chan, 2006; Liu, 2002). Indeed, the husband may be a passive observer of the in-law conflict or a participant in his wife's battering.

This study mainly focuses on in-law conflict, which is more common than in-law abuse. Existing studies of in-law conflict as a risk factor for IPV have mainly used case studies or qualitative studies with small sample sizes, so generalizing their findings is difficult, and it is not possible to statistically test in-law conflict as a risk factor using these data. So far, there have been no published data to quantitatively test the correlation between in-law conflict and IPV. To fill this research gap, we attempted to quantify the levels of in-law conflict and to examine the correlates of in-law conflict and IPV against pregnant women in a cohort of Chinese pregnant women who visited antenatal clinics in Hong Kong.

\section{Materials and Method}

\section{Design and Sample}

The study was carried out in seven Obstetrics and Gynaecology Units of the Hospital Authority in Hong Kong between July 2005 and April 2006. 
The seven units served about $80 \%$ of the pregnant women who visited antenatal clinics throughout the territory. All patients who met the study criteria during the study period were invited to participate. The inclusion criteria were pregnant women who were aged 18 to 50 years, who gave their informed consent, and who were in the 36th week of their pregnancy at the time of recruitment. The participants were interviewed face-to-face by research nurses who were trained to conduct research interviews with abused pregnant women.

\section{Measures}

The Abuse Assessment Screen (AAS) (Soeken, McFarlane, Parker, \& Lominack, 1998) was used to identify cases of violence. It has been extensively used in many different health care settings and has demonstrated satisfactory psychometric properties internationally (Soeken et al., 1998) and locally (W Leung, Kung, Lam, Leung, \& Ho, 2002). The AAS consists of questions designed to elicit the history of violence against women within a stated period and the identification of the perpetrator. When used with pregnant women, the AAS addresses violence during the individual's lifetime, during the preceding year, and during her pregnancy. The Chinese AAS used in this study is a translation of the AAS that was developed with the permission of the original test constructors. The Chinese AAS addresses emotional and physical violence separately for all stated time periods (lifetime, the preceding-year and during pregnancy). This differs from the AAS which treats lifetime emotional and physical violence simultaneously while focusing on physical violence only for the other time periods. The decision to treat emotional and physical violence separately in the Chinese AAS was justified because previous studies have revealed a predominance of emotional abuse among Chinese abused women and many of them did not report physical violence (Chan, 2005; . C. Leung et al., 1999; Leung et al., 2002; Tiwari et al., 2005). The Chinese AAS has been validated and demonstrated satisfactory measurement accuracy for identifying IPV among women in the Chinese population (Tiwari et al., in press).

One question was used to measure the frequency of in-law conflict. The participants were asked at the postdelivery interview the number of incidents of conflict with parents-in-law in the previous 12 months. The responses included never, once, twice, 3 to 5 times, 6 to 10 times, 11 to 20 times, 20 times or more, and none in the past 12 months but it has happened before.

The demographic questionnaire was used to detect the demographic and socioeconomic characteristics of the participants and to estimate the correlates of IPV and in-law conflict. It was administered at the initial interview. 
The questionnaire included items asking for information about the respondent's and her spouse's age, the age difference between her and her spouse, her and her spouse's education level, her marital status, the number of years she had been married, the number of years she had lived in Hong Kong, the number of children she had, whether this was her first pregnancy and whether it had been planned, her and her spouse's work status, her income, whether she was dependent on social support, whether she and her spouse were alcoholic, and whether she or a family member received social security, were currently in debt, or had a chronic illness.

This questionnaire was administered at the initial interview (36th week of pregnancy) and at the 1-week postdelivery telephone interview. If a participant gave a positive response to any item in any interview, she was classified as belonging to the abused group. There is evidence that studies that ask about violence more than once at different time slots, say, during the third trimester in pregnancy, report higher prevalence rates (Gazmararian et al., 1996).

The study was approved by the institutional review board of the Hospital Authority of Hong Kong. Participation in the study was voluntary, informed consent was provided, and confidentiality of information was guaranteed. Once identified as abused, participants were encouraged to seek help and were provided with the necessary information for referral.

\section{Statistical Analyses}

The demographic characteristics of the abused and nonabused pregnant women were summarized and compared by the exact likelihood ratio $\chi^{2}$ test (for categorical data) and the exact Wilcoxon rank sum test (for continuous data). The prevalence of abuse in the previous 12 months with breakdown by emotional and physical abuse was reported, with exact $95 \%$ confidence intervals (CIs) obtained from a binomial distribution.

To examine the association of demographics (Group 1), socioeconomic status and history of chronic diseases (Group 2), and in-law conflict (Group 3) with pregnancy abuse in the preceding year, a structured multiphase logistic regression analysis was performed with sequential causal relationship among the variables (Lam, Fong, Lauder, \& Lam, 2002). Specifically, we hypothesized that the variables in Group 1 would affect the variables in Group 2, but not vice versa, and similarly that the variables in Groups 1 and 2 would affect those in Group 3, but not vice versa. Phase 1 of the analysis was a forward stepwise logistic regression of all variables in Group 1. In Phase 2, a forward stepwise logistic regression was performed on the Group 2 variables after forcefully entering those variables that were significant in 
the Phase 1 analysis. In Phase 3, a forward stepwise logistic regression was performed on the Group 3 variables after forcefully entering those variables that were significant in Phase 1 and those that were significant in Phase 2. The effects of the variables in Group 1 were examined in Phase 1, those in Group 2 were examined in Phase 2, and so on.

The Hosmer and Lemeshow (H-L) test was used to assess the goodness of fit of the logistic regression analysis. A nonsignificant result implies adequacy of the logistic model. The effects of demographics, socioeconomic status, and history of chronic disease on the experience of in-law conflict were examined by a similar structured multiphase logistic regression analysis consisting of three phases.

The nominal level of significance was taken as 5\%, and the Statistical Analysis System (SAS) version 9 was used for the statistical analysis.

\section{Results}

\section{Demographic Characteristics of Participants}

A total of 3,245 pregnant women attending antenatal clinics in the participating hospitals successfully participated in the study. The abused and nonabused groups were similar in terms of age, spouse's age difference, education level, number of years married, years of stay in Hong Kong, and spouse's unemployment. Significantly more abused pregnant women were financially dependent (either unemployed or a housewife), had a low family income, had not planned their pregnancy, received social security, were currently in debt, were dependent on social support, had a family member with a chronic illness, and were alcoholic (either the participant or her spouse). These may be risk factors that need to be tested through multiple regression analysis. First $x_{x}$ pregnancy seemed to be a protective factor associated with less abuse during pregnancy. Although the $p$ values showed that the abuse rate was higher among single, divorced, or separated participants, the size of each group was too small for any firm conclusions to be drawn (Table 1).

\section{Prevalence of Abuse and In-Law Conflict}

Pregnant women reported having been abused by their intimate partners at a rate of $9.1 \%(95 \% \mathrm{CI}=8.2 \%, 10.2 \%)$ in the preceding year, among whom $6.7 \%(95 \% \mathrm{CI}=5.8 \%, 7.6 \%)$ were abused emotionally and $2.5 \%$ $(95 \% \mathrm{CI}=2.0 \%, 3.1 \%)$ were physically or sexually abused. With regard to 
Table 1

Characteristics of Pregnant Women Surveyed

\begin{tabular}{|c|c|c|c|c|c|c|c|}
\hline & \multicolumn{2}{|c|}{ Nonabused } & \multicolumn{2}{|c|}{ Abused } & \multicolumn{2}{|c|}{ Total $(N=3,245)$} & \multirow{2}{*}{$\begin{array}{c}p \\
\text { Value }^{\mathrm{a}}\end{array}$} \\
\hline & & $n$ & $\%$ & $n$ & $\%$ & $n$ & \\
\hline Age $(M, S D)$ & 2,949 & $30.8(4.8)$ & 296 & $30.5(5.6)$ & 3,245 & $30.8(4.9)$ & .696 \\
\hline Education & .253 & & & & & & \\
\hline Missing & 3 & 0.1 & 0 & 0.0 & 3 & 0.1 & \\
\hline Primary 1 to 6 & 130 & 4.1 & 18 & 6.1 & 138 & 4.3 & \\
\hline Form 1 to 6 & 2,112 & 71.6 & 221 & 74.7 & 2,333 & 71.9 & \\
\hline University or above & 707 & 24.0 & 56 & 18.9 & 763 & 23.5 & \\
\hline Unemployment & 215 & 7.3 & 44 & 14.9 & 259 & 8.0 & $<.001$ \\
\hline Housewife & 1,071 & 36.3 & 126 & 42.6 & 1,197 & 36.9 & .035 \\
\hline No. of children $(M, S D)$ & 2,794 & $0.5(0.7)$ & 279 & $0.7(0.8)$ & 3,073 & $0.6(0.7)$ & $<.001$ \\
\hline $\begin{array}{l}\text { Spouse age difference } \\
\geq 10 \text { years }\end{array}$ & 354 & 12.0 & 39 & 13.2 & 393 & 12.1 & .501 \\
\hline Marital status & & 0.0 & & 0.3 & 1 & 0.9 & $<.001$ \\
\hline Missing & & & & & & & \\
\hline Married or cohabiting & 2,881 & 97.7 & 275 & 92.9 & 3,156 & 97.3 & \\
\hline Single & 59 & 2.0 & 12 & 4.1 & 71 & 2.2 & \\
\hline Divorce or separated & 8 & 0.2 & 8 & 2.7 & 16 & 0.5 & \\
\hline Widowed & 1 & 0.0 & 0 & 0.0 & 1 & 0.0 & \\
\hline $\begin{array}{l}\text { No. of years married } \\
\qquad(M, S D)\end{array}$ & 2,886 & $4.7(3.7)$ & 280 & $5.0(4.0)$ & 3,166 & $4.7(3.7)$ & .527 \\
\hline $\begin{array}{l}\text { Years of stay in Hong } \\
\text { Kong: } \leq 7 \text { years }\end{array}$ & 632 & 21.4 & 61 & 20.6 & 693 & 21.4 & .115 \\
\hline Family monthly income & & & & & & & $<.001$ \\
\hline Missing & 39 & 1.3 & 6 & 2.0 & 45 & 1.4 & \\
\hline$<\mathrm{HK} \$ 5,000$ & 127 & 4.3 & 42 & 14.2 & 169 & 5.3 & \\
\hline $\mathrm{HK} \$ 5,000$ to 10,000 & 426 & 14.4 & 58 & 19.5 & 484 & 14.9 & \\
\hline $\mathrm{HK} \$ 10,000$ to 20,000 & 872 & 29.5 & 68 & 23.0 & 940 & 29.0 & \\
\hline $\mathrm{HK} \$ 20,000$ to 40,000 & 974 & 33.0 & 75 & 25.4 & 1,049 & 32.4 & \\
\hline$>\mathrm{HK} \$ 40,000$ & 511 & 17.4 & 47 & 15.9 & 558 & 17.2 & \\
\hline First pregnancy & 1,500 & 50.9 & 115 & 38.9 & 1,615 & 49.8 & $<.001$ \\
\hline Unplanned pregnancy & 983 & 33.3 & 162 & 54.7 & 1,145 & 35.3 & $<.001$ \\
\hline Receiving social security & 91 & 3.1 & 31 & 10.5 & 122 & 3.8 & $<.001$ \\
\hline Indebtedness & 148 & 5.0 & 44 & 14.9 & 192 & 5.9 & $<.001$ \\
\hline $\begin{array}{l}\text { Dependence on social } \\
\text { support }\end{array}$ & & & & & & & $<.001$ \\
\hline Missing & 2 & 0.1 & 0 & 0.0 & 2 & 0.1 & \\
\hline None & 1,669 & 56.6 & 134 & 45.3 & 1,803 & 55.6 & \\
\hline Peer or family & 1,093 & 37.1 & 120 & 40.5 & 1,213 & 37.4 & \\
\hline Social services & 185 & 6.3 & 42 & 14.2 & 227 & 7.0 & \\
\hline Chronic illness & 541 & 18.3 & 76 & 25.7 & 617 & 19.0 & .005 \\
\hline
\end{tabular}


Table 1 (continued)

\begin{tabular}{|c|c|c|c|c|c|c|c|}
\hline & \multicolumn{2}{|c|}{ Nonabused } & \multicolumn{2}{|c|}{ Abused } & \multicolumn{2}{|c|}{ Total $(N=3,245)$} & \multirow{2}{*}{$\begin{array}{c}p \\
\text { Value }^{a}\end{array}$} \\
\hline & $\%$ & $n$ & $\%$ & $n$ & $\%$ & $n$ & \\
\hline Alcoholic & 158 & 5.4 & 28 & 9.5 & 186 & 5.7 & .008 \\
\hline Spouse alcoholic & 587 & 19.9 & 85 & 28.7 & 672 & 20.7 & $<.001$ \\
\hline Spouse age $(M, S D)$ & 2,945 & $34.5(6.4)$ & 291 & $34.4(7.2)$ & 3,236 & $34.5(6.4)$ & .536 \\
\hline Spouse education & & & & & & & .068 \\
\hline Missing & 21 & 0.7 & 6 & 2.0 & 27 & 0.8 & \\
\hline None or kindergarten & 9 & 0.3 & 0 & 0.0 & 9 & 0.3 & \\
\hline Primary 1 to 6 & 116 & 3.9 & 23 & 7.8 & 139 & 4.3 & \\
\hline Form 1 to 6 & 1,994 & 67.6 & 195 & 65.9 & 2,189 & 67.4 & \\
\hline University or above & 809 & 27.4 & 72 & 24.3 & 881 & 27.1 & \\
\hline Spouse unemployed & 23 & 0.8 & 5 & 1.7 & 28 & 0.9 & .171 \\
\hline In-law conflict (lifetime) & 232 & 9.1 & 44 & 18.3 & 276 & 9.9 & $<.001$ \\
\hline $\begin{array}{l}\text { In-law conflict } \\
\text { (preceding year) }\end{array}$ & 152 & 6.0 & 35 & 14.5 & 187 & 6.7 & $<.001$ \\
\hline
\end{tabular}

Note: Values are $n$ and $\%$ unless otherwise noted.

a. By exact Wilcoxon rank sum test for age, no. of children, no. of years married, and spousal age and by exact likelihood ratio $\chi^{2}$ test for the others.

in-law conflict, about $9.9 \%(95 \% \mathrm{CI}=8.8 \%, 11.1 \%)$ and $6.7 \%(95 \% \mathrm{CI}=$ $5.8 \%, 7.7 \%$ ), respectively, reported having conflict with parents-in-law in their lifetime and in the preceding year. Significantly more abused pregnant women were having conflict with parents-in-law in their lifetime and in the preceding year.

\section{Factors Associated With Preceding-Year Abuse Among Pregnant Women}

After multivariate adjustment for covariates by a structured multiphase logistic regression analysis, in-law conflict, chronic illness, dependence on social support, unplanned pregnancy, first pregnancy, number of children, receiving social security, indebtedness, alcoholic, and education were strongly associated with preceding year abuse among the sampled pregnant women. The odd ratios are shown in Table 2. The value of Nagelkerke $R^{2}$ is $8 \%$, which indicates the proportion of variance explained. The result of the H-L test is 0.775 , which shows that the model was adequate. 
Table 2

Structured Multiphase Analysis of Preceding-Year IPV

\begin{tabular}{lccr}
\hline Variable & Adjusted $\mathrm{OR}^{\mathrm{a}}$ & $95.0 \%$ CI for OR & $p$ Value \\
\hline${\text { Phase } 1^{\mathrm{b}}}^{\mathrm{a}}$ & & & \\
Education & 0.87 & $0.79,0.97$ & .010 \\
Phase $2^{\mathrm{c}}$ & & & \\
Alcoholic & 1.88 & $1.09,3.23$ & .023 \\
Indebtedness & 2.65 & $1.67,4.20$ & $<.001$ \\
Receiving social security & 2.42 & $1.30,4.52$ & .005 \\
Number of children & 1.23 & $1.04,1.44$ & .015 \\
First pregnancy & 0.70 & $0.52,0.96$ & .026 \\
Unplanned pregnancy & 1.87 & $1.38,2.52$ & $<.001$ \\
Dependence on social support & 1.41 & $1.05,1.89$ & .024 \\
Chronic illness & 1.94 & $1.11,3.39$ & .020 \\
Phase $3^{\mathrm{d}}$ & & & \\
In-law conflict & 1.41 & $1.21,1.64$ & $<.001$ \\
\hline
\end{tabular}

Note: $\mathrm{OR}=$ odds ratio $\mathrm{CI}=$ confidence interval.

a. Variables in Phase 1 were adjusted by other significant variables in Phase 1. Variables in Phase 2 were adjusted by variables significant in Phase 1 or 2 . Variables in Phase 3 were adjusted by variables significant in Phase 1 or 2 or 3 .

b. $n=3,215$, Nagelkerke $R^{2}=.5 \%$, Hosmer \& Lemeshow test $p=.973$.

c. $n=2,472$, Nagelkerke $R^{2}=8 \%$, Hosmer \& Lemeshow test $p=.775$.

d. $n=2,336$, Nagelkerke $R^{2}=8 \%$, Hosmer \& Lemeshow test $p=.930$.

\section{Factors Associated With In-Law Conflict}

After we controlled for other factors with the multiple logistic regression models, chronic illness was strongly associated with in-law conflict that had occurred either in the participant's lifetime or in the preceding year. The odd ratios are shown in Tables 3 and 4. Education and first pregnancy were factors that reduced the level of in-law conflict. Indebtedness was strongly associated with lifetime in-law conflict, whereas alcoholism was strongly associated with in-law conflict in the preceding year. The values of Nagelkerke $R^{2}$ are $5 \%$ to $6 \%$, which indicates the proportion of variance explained. The results of the $\mathrm{H}-\mathrm{L}$ test are 0.883 and 0.595 , which show that the models were adequate.

\section{Discussion}

\section{Prevalence}

The prevalence of preceding-year violence against pregnant women was $9 \%$. This figure is comparable to that found with data gathered in studies of 
Table 3

Structured Multiphase Analysis of Lifetime In-Law Conflict

\begin{tabular}{lccr}
\hline Variable & Adjusted $\mathrm{OR}^{\mathrm{a}}$ & $95.0 \%$ CI for OR & $p$ Value \\
\hline Phase $1^{\mathrm{b}}$ & & & .002 \\
Education & 0.84 & $0.76,0.94$ & \\
Phase $2^{\mathrm{c}}$ & & & .028 \\
Indebtedness $_{\text {First pregnancy }}$ & 1.68 & $1.06,2.67$ & $<.001$ \\
Chronic illness & 0.46 & $0.35,0.62$ & $<.001$ \\
\hline
\end{tabular}

Note: $\mathrm{OR}=$ odds ratio $; \mathrm{CI}=$ confidence interval.

a. Variables in Phase 1 were adjusted by other significant variables in Phase 1. Variables in Phase 2 were adjusted by variables significant in Phase 1 or 2 .

b. $n=2,774$, Nagelkerke $R^{2}=.8 \%$, Hosmer \& Lemeshow test $p=.242$.

c. $n=2,555$, Nagelkerke $R^{2}=6 \%$, Hosmer \& Lemeshow test $p=.883$.

\section{Table 4}

Structured Multiphase Analysis Of Preceding-Year In-Law Conflict

\begin{tabular}{lccr}
\hline Variable & Adjusted $\mathrm{OR}^{\mathrm{a}}$ & $95.0 \% \mathrm{CI}$ for OR & $p$ Value \\
\hline${\text { Phase } 1^{\mathrm{b}}}^{\mathrm{b}}$ & & & .002 \\
Education & 0.84 & $0.76,0.94$ & \\
Phase $2^{\mathrm{c}}$ & & & .043 \\
Alcoholic & 1.81 & $1.02,3.21$ & $<.001$ \\
First pregnancy & 0.51 & $0.36,0.73$ & $<.001$ \\
Chronic illness & 2.46 & $1.75,3.46$ & \\
\hline
\end{tabular}

Note: $\mathrm{OR}=$ odds ratio $; \mathrm{CI}=$ confidence interval.

a. Variables in Phase 1 were adjusted by other significant variables in Phase 1. Variables in Phase 2 were adjusted by variables significant in Phase 1 or 2 .

b. $n=2,774$, Nagelkerke $R^{2}=.8 \%$, Hosmer \& Lemeshow test $p=.242$.

c. $n=2,555$, Nagelkerke $R^{2}=5 \%$, Hosmer \& Lemeshow test $p=.595$.

obstetric gynecology populations in which the rates of IPV during pregnancy ranged from $4 \%$ to $36 \%$ (C Leung et al., 1999; Wiemann, Agurcia, Berenson, Volk, \& Rickert, 2000). The majority of the victims were abused emotionally. Only $2 \%$ were physically or sexually abused by their intimate partners.

The lifetime prevalence rate of conflict with parents-in-law was $10 \%$, and the rate of preceding year in-law conflict was $7 \%$. These rates are comparable to the prevalence rates of abuse during pregnancy, and there was a significant correlation between in-law conflict and violence against pregnant women. 


\section{Risk Factors}

Unlike in some other studies in which age (Martin, Mackie, Kupper, Buescher, \& Moracco, 2001), unmarried status (Lipsky, Holt, Easterling, \& Critchlow, 2005), and unemployment (-. Leung et al., 1999) were found to be risk factors for violence against pregnant women, this was not confirmed in the current study. Consistent with other studies of pregnancy-related violence, a low level of education - which is an indicator of low socioeconomic status-being dependent on social support, having an unplanned pregnancy, and alcoholism were found to be risk factors for violence against pregnant women in a Chinese population. The number of children could also be a risk factor for violence. First $x_{\alpha}$ pregnancies were found to involve less violence. This was consistent with an earlier study that found that having a first child was significantly associated with a greater chance of violence cessation (Jasinski, 2001). However, it might only be a short-term cessation of violence, especially if there are no changes in other risk factors. A pregnant woman was at higher risk for partner violence if she or a family member had financial problems such as debt or a low income that necessitated receiving social security. This is consistent with Bullock, Mears, Woodcock, and Record's (2001) findings that women who have more financial worries are more likely to suffer from domestic violence. Having a chronic illness, or having a family member with a chronic illness, was also significantly correlated with partner violence. Women are the primary caretakers in traditional Chinese families, so they are the first to take care of family members who have health problems. This is often the case even when they are pregnant.

After controlling for covariates, in-law conflict remained the variable with the strongest association with preceding-year abuse among pregnant women. This is the first study to provide empirical data that confirm the relationship between in-law conflict and violence against women. In explaining in-law conflict as a risk factor, Fernandez (1997) used the concept of "conflicting identities" to explain the change in status of the mother-in-law from being a victim when she was a young daughter-in-law to being a batterer when she becomes a mother-in-law. This highlights the change in power status that a woman experiences as she moves between family roles. However, it does not explain how this would become a risk factor for violence against women. In fact, in a traditional Chinese family, the power status of a woman does not change as she moves from being a daughter-in-law to a mother-in-law because she retains the structural subordinated identity of being a woman. She is subordinate to the man, who is the head of the family. According to Confucius, a woman must yield obedience to the instructions of a man and 
help to carry out his principles. When young, she must obey her father and elder brother; when married she must obey her husband; when her husband is dead, she must obey her son. It is very common for a mother-in-law to follow the will of her son by disciplining a wife who creates in-law conflict. Chinese women are socialized to identify with men and their interests. Consequently, they could be perpetrators of oppression in their roles as superiors in racial/ethnic, class, generational, and other hierarchies. Violence against women is a product of the interlocking systems of gender and generation hierarchies in the family (Fernandez, 1997).

Several risk factors for in-law conflict were identified in this study. After controlling for other factors with the multiple logistic regression models, chronic illness remained the variables that was strongly associated with inlaw conflict. Education and first pregnancy seemed to be protective factors that would reduce in-law conflict. A pregnant woman was at higher risk of in-law conflict if she or a family member had financial problems such as debt. This factor, indebtedness, consistently predicted IPV and in-law conflict. Women's use of alcohol was also consistently associated with IPV and in-law conflict. With these data, we were unable to determine whether this is a risk factor or a dysfunctional coping behavior after violence and conflict. Whichever is the case, the health of abused pregnant women deserves considerable attention from health professionals.

\section{Implications for Research and Violence Prevention}

Our findings underscore the need to obtain information on in-law conflict as a risk factor for IPV. IPV is not a standalone problem but correlates with other forms of violence against women, such as in-law conflict, under the same familial hierarchical structure that is deeply rooted in Chinese culture. It seems that in-law conflict is a common phenomenon among Chinese and some Asian cultures. The paucity of research on in-law conflict in other ethnic groups reflects that the influence of extended family members may be underestimated in cultures that emphasize individualism. It may be a universal phenomenon that is more explicit in collectivist societies such as Chinese ones. It would be worthwhile for further research to test the possibility that in-law conflict is a risk factor for IPV in different cultures.

With regard to the prevention of violence against women, violence against women by different family members and using various types of abuse should be taken into account in universal screening and risk assessment. Some organizations of health professionals (e.g., the American College of Obstetricians and Gynecologists, 2002) have recommended screening women for domestic 
violence in health care settings. This should not be limited to IPV but should extend to all forms of violence against women. Risk assessment of IPV against pregnant women should also take into account alcohol use among pregnant women as well as stress, including stress caused by financial problems or chronic illness. Psychosocial support programs should be provided for pregnant women who have a low level of education or a low socioeconomic status. The perpetrators of partner violence and in-law conflict should also have access to such programs. Family-based interventions such as home visitations may also be appropriate (Olds et al., 1999).

A major limitation of this study is that in-law conflict was measured by a single item that asked participants the number of incidents of conflict with their parents-in-law during the previous 12 months. This may have led to an underestimation of the prevalence of in-law conflict because the term conflict may be subject to different interpretations. Other forms of abuse should be studied and used as a checklist when assessing in-law conflict. Some items related to in-law emotional abuse have recently been explored, such as isolation, social and economic control, domestic servitude, awareness or support of IPV, and direct physical abuse (Raj et al., 2006). Another concern is that in-law abuse was not measured in this study. The AAS as well as the Chinese AAS did ask who the perpetrators of violence against pregnant women were, including husband, ex-husband, boyfriend, stranger and others. The responses did not include in-laws. It would be worthwhile for further research to test the possibility of including in-law as one of the potential perpetrator of violence against pregnant women. More research is needed to further explore the construct of in-law abuse and conflict.

The prevalence of assault, which was measured by the AAS, could also have been underestimated because of the limited number of items and because, again, the term abuse may be subject to different interpretations (Jasinski, 2004; Reichenheim \& Moraes, 2004). However, the AAS is still the most commonly used measure of violence in research using clinical samples because of the ease of administration and the established reliability and validity of the scale (Jasinski, 2004).

Despite these limitations, this study provides preliminary evidence that in-law conflict is a risk factor for IPV. Even though relationship harmony is valued in Chinese tradition, some pregnant women in Hong Kong are abused by their intimate partners. Certain women, in particular those who have conflicts with their in-laws, appear to be at an especially high risk. The results have important implications for future research and violence prevention in health settings with regard to routine screening and family-based interventions. 


\section{References}

American College of Obstetricians and Gynecologists. (2002). Guidelines for women's health care (2nd ed.). Washington, DC: Author.

Bullock, L. F., Mears, J. L., Woodcock, C., \& Record, R. (2001). Retrospective study of the association of stress and smoking during pregnancy in rural women. Addictive Behaviors, 26(3), 405-413.

Chan, K. L. (2005). Study on child abuse and spouse battering: Report on findings of household survey. Hong Kong: University of Hong Kong, Department of Social Work \& Social Administration.

Chan, K. L. (2006). The Chinese concept of face and violence against women. International Social Work, 49(1), 65-73.

Counts, D. A., Brown, J. K., \& Campbell, J. (Eds.). (1999). To have and to hit: Cultural perspectives on wife beating ( $2 \mathrm{nd}$ ed.). Chicago: University of Illinois Press.

Dasgupta, S. D. (2000). Charting the course: An overview of domestic violence in the South Asian community in the United States. Journal of Social Distress and the Homeless, 9(3), 173-185.

Fernandez, M. (1997). Domestic violence by extended family members in India: Interplay of Gender and generation. Journal of Interpersonal Violence, 12, 433-455.

Gazmararian, J. A., Lazorick, S., Spitz, A. M., Ballard, T. J., Saltzman, L. E., \& Marks, J. S. (1996). Prevalence of violence against pregnant women. Journal of the American Medical Association, 275, 1915-1920.

Jasinski, J. L. (2001). Pregnancy and violence against women: An analysis of longitudinal data. Journal of Interpersonal Violence, 16, 712-733.

Jasinski, J. L. (2004). Pregnancy and domestic violence: A review of the literature. Trauma, Violence, \& Abuse, 5(1), 47-64.

Lam, C. L. K., Fong, D. Y. T., Lauder, I. J., \& Lam, T. P. (2002). The effect of health-related quality of life (HRQOL) on health service utilisation of a Chinese population. Social Science and Medicine, 55, 1635-1646.

Lee, D. T. S., Yip, A. S. K., Leung, T. Y. S., \& Chung, T. K. H. (2004). Ethnoepidemiology of postnatal depression: Prospective multivariate study of sociocultural risk factors in a Chinese population in Hong Kong. British Journal of Psychiatry, 184(1), 34-40.

Leung, T. W., Ng, E. H. Y., Leung, W. C., \& Ho, P. C. (2003). Intimate partner violence among infertile women. International Journal of Gynaecology and Obstetrics, 83, 323-324.

Leung, W. C., Kung, F., Lam, J., Leung, T. W., \& Ho, P. C. (2002). Domestic violence and postnatal depression in a Chinese community. International Journal of Gynaecology and Obstetrics, 79, 159-166.

Leung, W. C., Leung, T. W., Lam, Y. Y., \& Ho, P. C. (1999). The prevalence of domestic violence against pregnant women in a Chinese community. International Journal of Gynaecology and Obstetrics, 66, 23-30.

Lipsky, S., Holt, V. L., Easterling, T. R., \& Critchlow, C. W. (2005). Police-reported intimate partner violence during pregnancy: Who is at risk? Violence and Victims, 20(1), 69-86.

Liu, M. (2002). Rebellion and revenge: The meaning of suicide of women in rural China. International Journal of Social Welfare, 11, 300-309.

Martin, S. L., Mackie, L., Kupper, L. L., Buescher, P. A., \& Moracco, K. E. (2001). Physical abuse of women before, during, and after pregnancy. Journal of the American Medical Association, 285, 1581-1584. 
Olds, D. L., Henderson, C. R., Kitzman, H. J., Eckenrode, J. J., Cole, R. E., \& Tatelbaum, R. C. (1999). Prenatal and infancy home visitation by nurses: Recent findings. Future Child, 9(1), 44-65.

Raj, A., Livramento, K. N., Santana, M. C., Gupta, J., \& Silverman, J. G. (2006). Victims of intimate partner violence more likely to report abuse from in-laws. Violence Against Women, 12, 936-949.

Ramanathan, S. (1996). Violence against women. International Medical Journal, 3(2), 145-148.

Reichenheim, M. E., \& Moraes, C. L. (2004). Comparison between the Abuse Assessment Screen and the Revised Conflict Tactics Scales for measuring physical violence during pregnancy. Journal of Epidemiology and Community Health, 58, 523-527.

Rianon, N. J., \& Shelton, A. J. (2003). Perception of spousal abuse expressed by married Bangladeshi immigrant women in Houston. Journal of Immigrant Health, 5(1), 37-44.

Soeken, K. L., McFarlane, J., Parker, B., \& Lominack, M. C. (1998). The Abuse Assessment Screen: A clinical instrument to measure frequency, severity, and perpetrator of abused against women. In J. Campbell (Ed.), Empowering survivors of abuse: Health care for battered women and their children (pp. 195-203). Thousand Oaks, CA: Sage.

Tiwari, A., Fong, D. Y. T., Chan, K. L., Leung, W. C., Parker, B., \& Ho, P. C. (im press). Identify.ng intimate partner violence: Comparing the Chinese Abuse Assessment Screen with the Revised Conflict Tactics Scales. British Joumal of Obstetrics and Gynaecology.

Tiwari, A., Leung, W. C., Leung, T. W., Humphreys, J., Parker, B., \& Ho, P. C. (2005). A randomised controlled trial of empowerment training for Chinese abused pregnant women in Hong $\mathrm{H} \equiv$ British Jomal of Obstetries and Gyocolog, 112, 1249-1256.

Wiemann, $\square .$, Ag ircia, C. A., Berenson, A. B., Volk, R. J., \& Rickert, V. I. (2000). Pregnant adolescents: Experiences and behaviors associated with physical assault by an intimate partner. Maternal and Child Health Journal, 4(2), 93-101.

Xu, X., Zhu, F., O’Campo, P., Koenig, M. A., Mock, V., \& Campbell, J. (2005). Prevalence of and risk factors for intimate partner violence in China. American Journal of Public Health, 95(1), 78-85.

Yick, A. G. (1999). Domestic violence in the Chinese American community: Cultural taboos and barriers. Family Violence \& Sexual Assault Bulletin, 15(4), 16-23.

Ko Ling Chan is wit $\equiv$ Department of Social Work and Social Administration, University of Hong Kong.

Agnes Tiwari is with the Department of Nursing Studies, University of Hong Kong.

Daniel Fong is with the Department of Nursing Studies, University of Hong Kong.

Wing Cheong Leung is with the Department of Obstetrics and Gynaecology, Kwong Wah Hospital.

$$
\text { with }
$$

Douglas A. Brownridge is associate professor in the Department of Family Social Sciences, Arthur V. Mauro Centre for Peace and Justice, University of Manitoba, Canada.

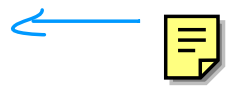

